\title{
Does dual-task training improve spatiotemporal gait parameters in Parkinson's disease?
}

\author{
Christian Geroin $^{\mathrm{a}, *}$, Jorik Nonnekes ${ }^{\mathrm{b}}$, Nienke M. de Vries ${ }^{\mathrm{c}}$, Carolien Strouwen ${ }^{\mathrm{d}, \mathrm{e}}$, Nicola Smania ${ }^{\mathrm{a}, \mathrm{f}}$, \\ Michele Tinazzi $^{\mathrm{g}}$, Alice Nieuwboer ${ }^{\mathrm{d}}$, Bastiaan R. Bloem ${ }^{\mathrm{c}}$ \\ ${ }^{a}$ Neuromotor and Cognitive Rehabilitation Research Center (CRRNC), Department of Neurosciences, Biomedicine and Movement Sciences, University of Verona, Verona, \\ Italy \\ ${ }^{\mathrm{b}}$ Radboud University Medical Center, Donders Institute for Brain, Cognition and Behaviour, Department of Rehabilitation, Nijmegen, The Netherlands \\ ${ }^{\mathrm{c}}$ Radboud University Medical Center, Donders Institute for Brain, Cognition and Behaviour, Department of Neurology, Nijmegen, The Netherlands \\ ${ }^{\mathrm{d}}$ Department of Rehabilitation Sciences, Faculty of Kinesiology and Rehabilitation, KU Leuven, Leuven, Belgium \\ ${ }^{\mathrm{e}}$ Rehabilitation Research Center (REVAL), Biomedical Research Institute (BiOMED), Faculty of Medicine and Life Sciences, Hasselt University, Belgium \\ ${ }^{\mathrm{f}}$ Neurorehabilitation Unit, Azienda Ospedaliera Universitaria Integrata, Verona, Italy \\ ${ }^{g}$ Neurology Unit, Movement Disorders Division, Department of Neurosciences, Biomedicine and Movement Sciences, University of Verona, Verona, Italy
}

\section{A R T I C L E I N F O}

\section{Keywords:}

Parkinson's disease

Gait

Postural control

Dual-task

Physical therapy

\begin{abstract}
A B S T R A C T
Introduction: The DUALITY trial recently showed that both integrated and consecutive dual-task training improve dual-task gait velocity, without increasing fall risks in patients with Parkinson's disease (PD). Gait velocity was the primary outcome; not reported, however, were important gait measures related to the risk of falling such as gait variability. In this secondary analysis, we compared the efficacy of the two training programs with respect to spatiotemporal outcome parameters.

Methods: 121 PD patients (Hoehn and Yahr stage II-III while ON medication) were randomly assigned to either a consecutive group $(n=65)$ in which cognitive and gait tasks were trained separately, or an integrated group $(n=56)$ in which cognitive and gait tasks were trained simultaneously. Both groups received 24 in-home physiotherapy sessions for six consecutive weeks. Two baseline measurements were performed during a six-week control period prior to the interventions. Gait was evaluated under three different (and untrained) dual-task conditions immediately after the treatment period and at 12-week follow-up.

Results: Both training modalities had a comparable effect on spatiotemporal gait parameters. A significant posttraining increase in stride length $(\mathrm{P}<.001)$ and cadence $(\mathrm{P}<.001)$ was found under both the single and the dual-task conditions. These improvements were maintained at follow-up, although the effect was slightly reduced. No significant changes were found for gait variability under single and dual-task conditions.

Conclusion: We found both integrated and consecutive dual-task training to be safe and effective in improving several spatiotemporal gait parameters under trained and untrained dual-task conditions.
\end{abstract}

\section{Introduction}

Gait impairments are common in Parkinson's disease (PD) and involve - but are not limited to -reduced stride length, slower gait velocity, and increased gait variability [1-3]. Dual-task performance is affected in PD, with a worsening of spatiotemporal gait parameters when walking is combined with a secondary task [4]. In addition, patients may experience freezing events as the complexity of concurrent tasks increases [5,6]. Freezing, in turn, is a major risk factor for falls
[7].

Compromised dual-task performance during gait has disabling consequences for daily life activities. Indeed, impaired dual-task performance has been shown to correlate with an increased risk of falling [4], reduced functional abilities [8], and diminished quality of life [4]. Evidence indicates that physiotherapy can improve dual-task performance during gait, but questions remain about which type of training should be applied. The DUALITY trial was designed to explore two different approaches: integrated dual-task training (IDT) for the

\footnotetext{
* Corresponding author. Neuromotor and Cognitive Rehabilitation Research Center (CRRNC), Department of Neurosciences, Biomedicine and Movement Sciences, University of Verona, P.le L.A. Scuro 10, 37134 Verona, Italy.

E-mail addresses: christian.geroin@univr.it (C. Geroin), Jorik.Nonnekes@radboudumc.nl (J. Nonnekes), Nienke.deVries-Farrouh@radboudumc.nl (N.M. de Vries), carolien.strouwen@gmail.com (C. Strouwen), nicola.smania@univr.it (N. Smania), michele.tinazzi@univr.it (M. Tinazzi), Alice.Nieuwboer@kuleuven.be (A. Nieuwboer), Bas.Bloem@radboudumc.nl (B.R. Bloem).
} 
simultaneous practice of cognitive and gait tasks, and consecutive task training (CCT) for separate training of cognitive and gait tasks [9]. The recently published results of the DUALITY trial showed improvement in dual-task gait velocity after both training approaches, without increasing the risk of falls quantified for four 6-week periods [10] as measured using weekly phone calls to study participants. They were accompanied by significant improvements in such cognitive domains as reaction time and percentage of corrected responses on the stroop test dual task [10].

In the original analysis of the DUALITY trial, gait velocity during dual-tasking was the predefined primary outcome, but the effects on the other spatiotemporal gait parameters during dual-tasking were not reported. But because gait velocity is not the only gait parameter affected during dual-task gait performance in PD [4], it is unclear whether gait velocity increased with improved stride length or was due to a positive change in cadence. Moreover, it is unclear whether gait variability - a measure closely associated with the risk of falling [2,3] - changed because of dual-task training.

In this secondary analysis, we compared the efficacy of the two dual-task training programs in improving spatiotemporal gait parameters, with a focus on stride length, cadence, and gait variability in particular. As the primary analysis showed improvement in gait speed after IDT and CTT [10], we hypothesized that the training programs would also improve stride length, cadence, and gait variability.

\section{Methods}

\subsection{Study design and participants}

The outline of the DUALITY trial has been published elsewhere $[9,10]$. In summary, the DUALITY trial is a single-blind, randomized, controlled trial (RCT) in which data were collected at two study sites between March 2012 and December 2014. The study was approved by the ethical committees of the University Hospitals Leuven (Belgium) and the Radboud University Medical Center Nijmegen (the Netherlands). All participants signed an informed consent form before participation.

Inclusion criteria were: (1) a diagnosis of PD according to the UK Brain Bank criteria [11], (2) Hoehn and Yahr (H\&Y) stages II-III when on medication [12], (3) ability to walk 10 min continuously, (4) presence of dual-task (DT) interference as established by a structured checklist [9], (5) Mini-Mental State Examination score $\geq 24$ [13], (6) stable medication over the past 3 months, (7) no hearing or visual problems that could interfere with testing or training, and (8) no deep brain stimulator or stable deep brain stimulator settings during the past year. Exclusion criteria were any unstable other medical conditions that affected gait. Details on inclusion criteria, randomization process, and flow diagram of the study are given elsewhere $[9,10]$.

\subsection{Interventions}

Trained physiotherapists delivered in-home treatment sessions twice a week to both groups for 6 consecutive weeks. The training sessions comprised $30 \mathrm{~min}$ of gait and cognitive exercises plus $10 \mathrm{~min}$ of functional practice. In addition, patients performed an unsupervised exercise program twice a week that included gait training and mental practice using an MP3 player (30 min per session) [9]. The exact contents of the supervised and the unsupervised exercises differed between the CTT and IDT group, as described elsewhere [9]. In brief, dual-tasks were avoided in the CTT group, and gait and cognitive task were delivered separately whenever possible [9]. In contrast, the IDT group patients performed the same cognitive tasks and gait exercises simultaneously. Cognitive exercise difficulty was increased progressively in both groups. Five categories of exercises were presented: verbal fluency, discrimination and decision-making tasks, working memory tasks, mental tracking tasks, and reaction time tasks. Flawless performance on a given task was prerequisite for progression to the next level of task difficulty.

\subsection{Outcomes}

A blinded physiotherapist tested the patients during ON-medication at four time points: the start (test 1) and the end (test 2) of a 6-week baseline period; immediately after the 6-week training period (test 3); and after 12 weeks of follow-up without training (test 4). Spatiotemporal gait parameters, including stride length $(\mathrm{cm})$, cadence (step/min), standard deviation of stride length $(\mathrm{cm})$, standard deviation of stride time (s), stride time (stride/sec), swing \% (\% of gait cycle), swing time (sec), stance \% (\% of gait cycle), stance time (sec), double support \% (\% of gait cycle), support base (cm), and step length asymmetry $(\mathrm{cm})$ were measured using a GAITRite walkway system (CIR Systems Inc, Havertown, PA). The system was $7.92 \mathrm{~m}$ in length and operated at a sampling frequency of $120 \mathrm{~Hz}$. Gait variability was evaluated by comparing 1) stride length variability and 2) stride time variability. We used the within-person standard deviation instead of the coefficient of variation because it has better reliability [14].

Patients were asked to walk without secondary tasks at a comfortable walking speed. Gait parameters were also evaluated in three types of dual-task conditions: 1) an auditory stroop task; 2) a backward digit span task; and 3) while using a mobile phone [9]. The backward digit span task was trained, while the auditory stroop task and use of the mobile phone were not trained during the training period. The single and the dual-task test order was randomized but kept constant for each patient throughout the trial.

\subsection{Statistical analysis}

Statistical analysis was performed according to the intention-totreat principle and included all individuals initially enrolled in the study. Missing data (2-13\%) were due to drop-out (6.6\%), human errors, and technical problems [10]. We used linear mixed models (a direct likelihood estimation technique) to analyze all continuous outcomes as values were missing at random. Two-sided p-values were set at an $\alpha$ level of 0.05 . Fixed factors were treatment group (IDT vs. CTT), time (test 1-4), and the interaction between Time by Group. Participants were included as random factor. Bonferroni's correction was applied for multiple comparisons. The descriptive statistics included means, standard deviations, and 95\% confidence intervals. Statistical analysis was performed with SPSS 20.0 (SPSS Inc, Chicago, IL, USA).

\section{Results}

A total of 121 patients were randomized to receive either CCT $(n=65)$ or IDT $(n=56)$. The dropout rate was 9.9\%: 4 participants dropped out of CTT and 8 dropped out of IDT. The reasons were generally health or time constraints and unrelated to the nature of the intervention. The participants who dropped out were not significantly different from those who completed the study. No adverse events were reported during the study period. The participants differed by level of education but the groups were similar for all clinical and demographical characteristics, including levodopa equivalent daily dose (LEDD) (Table 1). LEDD was entered as covariate in the statistical analysis because it was not stable over time ( $\mathrm{F}=7.41, \mathrm{p}<.001)$, with higher doses reported at test 3 and 4 [10].

No interaction effects between Time and Group were found for any of the spatiotemporal gait parameters (Tables 2 and 3 and Supplementary Tables 1 and 2), which indicates that both training modalities had similar effects.

\subsection{Stride length}

The main effects for time were significant for stride length in the 
Table 1

Clinical and demographical variables of patients at baseline 1 .

\begin{tabular}{|c|c|c|c|c|}
\hline & $\begin{array}{l}\text { Total group } \\
\mathrm{N}=121\end{array}$ & $\begin{array}{l}\text { CCT } \\
N=65\end{array}$ & $\begin{array}{l}\text { IDT } \\
N=56\end{array}$ & $\begin{array}{l}\text { CTT vs. } \\
\text { IDT } \\
\text { P-Value }\end{array}$ \\
\hline Age (year) & $65.93(9.22)$ & $66.05(9.30)$ & $65.80(9.19)$ & n.s. \\
\hline Gender $(\mathrm{M} / \mathrm{F})$ & $88 / 33$ & $49 / 16$ & $39 / 17$ & n.s. \\
\hline Disease duration (years) & $8.67(5.83)$ & $8.89(6.30)$ & $8.41(5.29)$ & n.s. \\
\hline $\mathrm{H} \& \mathrm{Y}$ (mean, SD) & $2.30(0.51)$ & $2.31(0.53)$ & $2.29(0.49)$ & n.s. \\
\hline Freezing of gait (yes/no) & $68 / 53$ & $34 / 31$ & $34 / 22$ & n.s. \\
\hline $\begin{array}{l}\text { Recurrent falls in the } 6 \\
\text { months prior to the } \\
\text { study (Yes/No) }\end{array}$ & $40 / 81$ & $22 / 43$ & $18 / 38$ & n.s. \\
\hline LEDD (mg/day) & $\begin{array}{l}687.80 \\
(431.66)\end{array}$ & $\begin{array}{l}752.25 \\
(453.24)\end{array}$ & $\begin{array}{l}612.99 \\
(396.10)\end{array}$ & n.s. \\
\hline MMSE $(/ 30)$ & 27.94 (1.59) & $27.88(1.65)$ & $28.02(1.53)$ & n.s. \\
\hline \multicolumn{5}{|l|}{ Level of education (no.) } \\
\hline 1 & 19 & 13 & 6 & \\
\hline 2 & 37 & 21 & 16 & n.s. \\
\hline 3 & 47 & 23 & 24 & \\
\hline 4 & 18 & 8 & 10 & \\
\hline
\end{tabular}

CTT, consecutive task training group; IDT, integrated dual task training group; $\mathrm{n}$, number of patients; H\&Y, Hoehn \& Yahr stage; LEDD, levodopa equivalent daily dose; MMSE, Mini-Mental State Examination; Level 1: schooling until age 15 (first 3 years of secondary school); Level 2: schooling until age 18 (secondary school); Level 3: higher education for 2, 3 or 4 years according to type of study after secondary school; Level 4: college and at least an extra 4 years of education after secondary school.

single-task conditions $(\mathrm{F}=23.06 ; \mathrm{P}<.001)$ and for all three $\mathrm{DT}$ conditions (Stroop $\mathrm{F}=30.64, \mathrm{P}<.001$; backward digit $\mathrm{F}=22.34$; $\mathrm{P}<.001 ;$ mobile phone $\mathrm{F}=13.73 ; \mathrm{P}<.001)$, indicating that $\mathrm{DT}$ stride length improved with both training modalities over baseline. Post hoc within-group comparisons showed significant increases in stride length after treatment and at follow-up in the single $(\mathrm{P}<.001)$, auditory stroop task $(\mathrm{P}<.001)$, backward digit span task $(\mathrm{P}<.001)$, and mobile phone task conditions $(\mathrm{P}<.001, \mathrm{P}<.006)$ (Tables 2 and 3 and Supplementary Tables 1 and 2; Fig. 1).

\subsection{Cadence}

The main effects for time were significant for cadence in the singletask $(\mathrm{F}=5.50, \mathrm{P}<.001)$ auditory stroop task $(\mathrm{F}=21.85 ; \mathrm{P}<.001)$, backward digit span task ( $\mathrm{F}=23.88$; $\mathrm{P}<.001)$, and mobile phone task condition $(\mathrm{F}=15.95$; $\mathrm{P}<.001)$. Post hoc within-group comparisons showed significant increase in cadence after treatment and at follow-up in the auditory stroop task $(\mathrm{P}<.001)$, backward digit task ( $\mathrm{P}<.001)$, but only at the follow-up for the mobile phone task condition $(\mathrm{P}<.001)$ (Tables 2 and 3 and Supplementary Tables 1 and 2; Fig. 1).

\subsection{Gait variability}

The main effects for time were significant for gait variability in terms of stride length variability on the backward digit span task $(\mathrm{F}=4.37 ; \mathrm{P}<.005)$ but not in the other conditions. Post hoc analysis revealed no significant differences on the backward digit span task. The main effects for time were only significant for 'stride time variability' in the auditory stroop task condition $(\mathrm{F}=4.50 ; \mathrm{P}<.005)$ but not in the other conditions. Again, post-hoc analysis disclosed no significant differences.

Table 2

Descriptive and inferential statistics for the auditory stroop task condition.

\begin{tabular}{|c|c|c|c|c|c|c|c|c|c|c|c|}
\hline \multirow[t]{2}{*}{ Outcomes } & \multirow[t]{2}{*}{ Group } & \multirow{2}{*}{$\begin{array}{l}\text { Baseline } \\
\text { Mean (SD) }\end{array}$} & \multirow{2}{*}{$\begin{array}{l}\text { Before } \\
\text { Treatment }\end{array}$} & \multirow{2}{*}{$\begin{array}{l}\text { After } \\
\text { Treatment }\end{array}$} & \multirow{2}{*}{$\begin{array}{l}\text { 12-Week FU } \\
\text { Mean (SD) }\end{array}$} & \multicolumn{3}{|c|}{ Linear Regression Model } & \multicolumn{3}{|c|}{ Post Hoc Analysis } \\
\hline & & & & & & Time & Group & Time*Group & $\begin{array}{l}\text { Baseline (P- } \\
\text { Value) }\end{array}$ & $\begin{array}{l}\text { After } \\
\text { (P-Value) }\end{array}$ & $\begin{array}{l}\text { FU } \\
\text { (P-Value) }\end{array}$ \\
\hline \multirow[t]{2}{*}{ Stride length (cm) } & CTT & $\begin{array}{l}111.89 \\
(18.38)\end{array}$ & $111.18(17.33)$ & $118.08(17.86)$ & $\begin{array}{l}114.59 \\
(20.69)\end{array}$ & $.001 \%$ & n.s. & n.s. & n.s. & $.001 *$ & $.001 *$ \\
\hline & IDT & $\begin{array}{l}110.22 \\
(19.20)\end{array}$ & $111.97(17.40)$ & $119.52(16.09)$ & $\begin{array}{l}119.31 \\
(17.63)\end{array}$ & & & & & & \\
\hline \multirow[t]{2}{*}{ Cadence (step/min) } & СTT & $\begin{array}{l}99.19 \\
(12.13)\end{array}$ & $99.88(10.16)$ & $103.86(10.34)$ & $\begin{array}{l}103.73 \\
(10.76)\end{array}$ & $.001 *$ & n.s. & n.s. & n.s. & $.001 *$ & $.001 *$ \\
\hline & IDT & $\begin{array}{l}99.19 \\
(12.32)\end{array}$ & $101.30(12.46)$ & 105.65 (10.19) & $\begin{array}{l}104.03 \\
(10.40)\end{array}$ & & & & & & \\
\hline \multirow[t]{2}{*}{ SD, stride length $(\mathrm{cm})$} & CTT & $5.35(2.54)$ & $5.35(4.20)$ & $5.43(3.26)$ & $5.03(2.61)$ & n.s. & n.s. & n.s. & n.s. & n.s. & n.s. \\
\hline & IDT & $5.47(2.62)$ & $5.18(3.50)$ & $5.15(3.06)$ & $4.72(2.15)$ & & & & & & \\
\hline SD, stride time (s) & $\begin{array}{l}\text { CTT } \\
\text { IDT }\end{array}$ & $\begin{array}{l}0.06(0.07) \\
0.06(0.04)\end{array}$ & $\begin{array}{l}0.05(0.07) \\
0.05(0.05)\end{array}$ & $\begin{array}{l}0.05(0.04) \\
0.04(0.05)\end{array}$ & $\begin{array}{l}0.04(0.03) \\
0.04(0.03)\end{array}$ & $.005 *$ & n.s. & n.s. & n.s. & n.s. & n.s. \\
\hline \multicolumn{12}{|c|}{ Other spatiotemporal gait parameters } \\
\hline Stride time (stride/sec) & $\begin{array}{l}\text { CTT } \\
\text { IDT }\end{array}$ & $\begin{array}{l}1.23(0.17) \\
1.23(0.15)\end{array}$ & $\begin{array}{l}1.21(0.13) \\
1.20(0.16)\end{array}$ & $\begin{array}{l}1.16(0.12) \\
1.15(0.12)\end{array}$ & $\begin{array}{l}1.17(0.13) \\
1.16(0.12)\end{array}$ & $.001 *$ & n.s. & n.s. & n.s. & $.001 *$ & $.001 *$ \\
\hline Swing \% (\% of gait cycle) & $\begin{array}{l}\text { CTT } \\
\text { IDT }\end{array}$ & $\begin{array}{l}33.57(2.62) \\
33.60(2.48)\end{array}$ & $\begin{array}{l}33.66(2.22) \\
33.81(2.54)\end{array}$ & $\begin{array}{l}34.50(2.27) \\
34.70(2.11)\end{array}$ & $\begin{array}{l}34.11(2.52) \\
34.61(2.40)\end{array}$ & $.001 *$ & n.s. & n.s. & n.s. & $.001 *$ & $.003^{*}$ \\
\hline Swing time (sec) & $\begin{array}{l}\text { CTT } \\
\text { IDT }\end{array}$ & $\begin{array}{l}0.41(0.05) \\
0.41(0.05)\end{array}$ & $\begin{array}{l}0.41(0.05) \\
0.41(0.06)\end{array}$ & $\begin{array}{l}0.40(0.05) \\
0.40(0.04)\end{array}$ & $\begin{array}{l}0.40(0.05) \\
0.40(0.05)\end{array}$ & $.001 *$ & n.s. & n.s. & n.s. & n.s. & n.s. \\
\hline Stance $\%$ (\% of gait cycle) & $\begin{array}{l}\text { CTT } \\
\text { IDT }\end{array}$ & $\begin{array}{l}66.45(2.61) \\
66.40(2.48)\end{array}$ & $\begin{array}{l}66.35(2.22) \\
66.20(2.54)\end{array}$ & $\begin{array}{l}65.51(2.27) \\
65.31(2.11)\end{array}$ & $\begin{array}{l}65.90(2.53) \\
65.40(2.40)\end{array}$ & $.001 \%$ & n.s. & n.s. & n.s. & $.001 *$ & $.002 *$ \\
\hline Stance time (sec) & $\begin{array}{l}\text { CTT } \\
\text { IDT }\end{array}$ & $\begin{array}{l}0.82(0.14) \\
0.82(0.11)\end{array}$ & $\begin{array}{l}0.81(0.09) \\
0.80(0.12)\end{array}$ & $\begin{array}{l}0.76(0.09) \\
0.75(0.09)\end{array}$ & $\begin{array}{l}0.77(0.09) \\
0.76(0.09)\end{array}$ & $.001 *$ & n.s. & n.s. & n.s. & $.001 *$ & $.001 *$ \\
\hline $\begin{array}{l}\text { Double support } \% \text { (\% of gait } \\
\text { cycle) }\end{array}$ & $\begin{array}{l}\text { CTT } \\
\text { IDT }\end{array}$ & $\begin{array}{l}32.74(5) \\
32.82(4.92)\end{array}$ & $\begin{array}{l}32.63(4.38) \\
32.39(4.75)\end{array}$ & $\begin{array}{l}30.98(4.36) \\
30.43(4.19)\end{array}$ & $\begin{array}{l}31.69(5.09) \\
30.85(4.62)\end{array}$ & $.001 *$ & n.s. & n.s. & n.s. & $.001 *$ & $.003^{*}$ \\
\hline Support base $(\mathrm{cm})$ & $\begin{array}{l}\text { CTT } \\
\text { IDT }\end{array}$ & $\begin{array}{l}11.96(3.42) \\
11.66(2.87)\end{array}$ & $\begin{array}{l}11.58(3.20) \\
11.44(2.83)\end{array}$ & $\begin{array}{l}11.50(3.11) \\
11.13(2.87)\end{array}$ & $\begin{array}{l}11.49(3.16) \\
11.06(2.58)\end{array}$ & $.002^{*}$ & n.s. & n.s. & $0.04 *$ & n.s. & n.s. \\
\hline Step length asymmetry $(\mathrm{cm})$ & $\begin{array}{l}\text { CTT } \\
\text { IDT }\end{array}$ & $\begin{array}{l}3.92(4.89) \\
3.26(2.35)\end{array}$ & $\begin{array}{l}3.64(3.15) \\
3.34(2.56)\end{array}$ & $\begin{array}{l}3.75(3.58) \\
3.34(2.04)\end{array}$ & $\begin{array}{l}3.76(2.90) \\
3.66(2.75)\end{array}$ & n.s. & n.s. & n.s. & n.s. & n.s. & n.s. \\
\hline
\end{tabular}

Abbreviations: CTT, consecutive task training group; IDT, integrated dual task training group; SD, standard deviation; FU, follow-up; * and in bold, statistically significant values. For linear regression model, $\mathrm{p}$ value significant if $<0.05$. Post-hoc analysis was performed only when time or group $\times$ time interactions were statistically significant. For post hoc analysis, P significant if $<.05$. 
Table 3

Descriptive and inferential statistics for the backwards digit span task condition.

\begin{tabular}{|c|c|c|c|c|c|c|c|c|c|c|c|}
\hline \multirow[t]{2}{*}{ Outcomes } & \multirow[t]{2}{*}{ Group } & \multirow{2}{*}{$\begin{array}{l}\text { Baseline } \\
\text { Mean (SD) }\end{array}$} & \multirow{2}{*}{$\begin{array}{l}\begin{array}{l}\text { Before } \\
\text { Treatment }\end{array} \\
\text { Mean (SD) }\end{array}$} & \multirow{2}{*}{$\begin{array}{l}\text { After } \\
\text { Treatment }\end{array}$} & \multirow{2}{*}{$\begin{array}{l}\text { 12-Week FU } \\
\text { Mean (SD) }\end{array}$} & \multicolumn{3}{|c|}{ Linear Regression Model } & \multicolumn{3}{|c|}{ Post Hoc Analysis } \\
\hline & & & & & & Time & Group & Time*Group & $\begin{array}{l}\text { Baseline (P- } \\
\text { Value) }\end{array}$ & $\begin{array}{l}\text { After } \\
\text { (P-Value) }\end{array}$ & $\begin{array}{l}\text { FU } \\
\text { (P-Value) }\end{array}$ \\
\hline \multirow[t]{2}{*}{ Stride length $(\mathrm{cm})$} & СTT & $\begin{array}{l}113.46 \\
(17.87)\end{array}$ & $112.62(16.88)$ & 119.14 (17.97) & $\begin{array}{l}117.63 \\
(18.70)\end{array}$ & $.001 *$ & n.s. & n.s. & n.s. & $.001 *$ & $.001 *$ \\
\hline & IDT & $\begin{array}{l}111.86 \\
(18.20)\end{array}$ & $112.84(17.28)$ & $119.48(16.43)$ & $\begin{array}{l}119.67 \\
(17.27)\end{array}$ & & & & & & \\
\hline \multirow[t]{2}{*}{ Cadence (step/min) } & СTT & $\begin{array}{l}95.54 \\
(12.81)\end{array}$ & $98.68(10.35)$ & $103.15(9.98)$ & $\begin{array}{l}102.32 \\
(10.28)\end{array}$ & $.001 *$ & n.s. & n.s. & $.001 *$ & $.001 *$ & $.001 *$ \\
\hline & IDT & $\begin{array}{l}97.70 \\
(13.18)\end{array}$ & $100.47(12.70)$ & $102.15(11.15)$ & $\begin{array}{l}103.02 \\
(11.20)\end{array}$ & & & & & & \\
\hline \multirow[t]{2}{*}{ SD, stride length $(\mathrm{cm})$} & CTT & $5.93(2.64)$ & $5.35(3.68)$ & $4.78(1.72)$ & $5.11(3.34)$ & $.005 *$ & n.s. & n.s. & n.s. & n.s. & n.s. \\
\hline & IDT & $6.43(4.47)$ & $5.84(4.76)$ & $5.17(3.07)$ & $5.05(2.62)$ & & & & & & \\
\hline SD, stride time (s) & $\begin{array}{l}\text { CTT } \\
\text { IDT }\end{array}$ & $\begin{array}{l}0.09(0.13) \\
0.08(0.09)\end{array}$ & $\begin{array}{l}0.05(0.04) \\
0.15(0.57)\end{array}$ & $\begin{array}{l}0.04(0.03) \\
0.11(0.45)\end{array}$ & $\begin{array}{l}0.06(0.13) \\
0.07(1.18)\end{array}$ & n.s. & n.s. & n.s. & n.s. & n.s. & n.s. \\
\hline \multicolumn{12}{|c|}{ Other spatiotemporal gait parameters } \\
\hline Stride time (stride/sec) & $\begin{array}{l}\text { CTT } \\
\text { IDT }\end{array}$ & $\begin{array}{l}1.28(0.20) \\
1.27(0.18)\end{array}$ & $\begin{array}{l}1.23(0.14) \\
1.24(0.23)\end{array}$ & $\begin{array}{l}1.17(0.12) \\
1.19(0.16)\end{array}$ & $\begin{array}{l}1.19(0.12) \\
1.18(0.14)\end{array}$ & $.001 *$ & n.s. & n.s. & n.s. & $.007^{*}$ & $.007^{*}$ \\
\hline Swing \% (\% of gait cycle) & $\begin{array}{l}\text { CTT } \\
\text { IDT }\end{array}$ & $\begin{array}{l}34.06(2.37) \\
33.88(2.57)\end{array}$ & $\begin{array}{l}33.83(2.11) \\
34.08(2.38)\end{array}$ & $\begin{array}{l}34.71(2.16) \\
34.83(2.14)\end{array}$ & $\begin{array}{l}34.60(2.58) \\
34.74(2.04)\end{array}$ & $.001 *$ & n.s. & n.s. & n.s. & $.001 *$ & $.001 *$ \\
\hline Swing time (sec) & $\begin{array}{l}\text { CTT } \\
\text { IDT }\end{array}$ & $\begin{array}{l}0.44(0.07) \\
0.43(0.05)\end{array}$ & $\begin{array}{l}0.42(0.05) \\
0.43(.16)\end{array}$ & $\begin{array}{l}0.41(0.04) \\
0.42(0.09)\end{array}$ & $\begin{array}{l}0.41(0.06) \\
0.41(0.05)\end{array}$ & $.004 *$ & n.s. & n.s. & n.s. & n.s. & n.s. \\
\hline Stance $\%$ (\% of gait cycle) & $\begin{array}{l}\text { CTT } \\
\text { IDT }\end{array}$ & $\begin{array}{l}65.95(2.37) \\
66.12(2.56)\end{array}$ & $\begin{array}{l}66.18(2.11) \\
65.96(2.40)\end{array}$ & $\begin{array}{l}65.29(2.17) \\
65.17(2.15)\end{array}$ & $\begin{array}{l}65.43(2.53) \\
65.27(2.05)\end{array}$ & $.001 *$ & n.s. & n.s. & n.s. & $.001 *$ & $.001 *$ \\
\hline Stance time (sec) & $\begin{array}{l}\text { CTT } \\
\text { IDT }\end{array}$ & $\begin{array}{l}0.85(.014) \\
0.84(0.15)\end{array}$ & $\begin{array}{l}0.81(0.10) \\
0.81(0.13)\end{array}$ & $\begin{array}{l}0.77(0.08) \\
0.77(0.09)\end{array}$ & $\begin{array}{l}0.78(0.09) \\
0.77(0.09)\end{array}$ & $.001 *$ & n.s. & n.s. & $.01 *$ & $.001 *$ & $.001 *$ \\
\hline $\begin{array}{l}\text { Double support } \% \text { (\% of gait } \\
\text { cycle) }\end{array}$ & $\begin{array}{l}\text { CTT } \\
\text { IDT }\end{array}$ & $\begin{array}{l}32.07(5.71) \\
32.13(5.20)\end{array}$ & $\begin{array}{l}32.21(4.28) \\
32.65(6.94)\end{array}$ & $\begin{array}{l}30.53(4.36) \\
31.03(5.40)\end{array}$ & $\begin{array}{l}31.19(5.10) \\
30.79(5.10)\end{array}$ & $.001 *$ & n.s. & n.s. & n.s. & $.001 *$ & $.03 *$ \\
\hline Support base $(\mathrm{cm})$ & $\begin{array}{l}\text { CTT } \\
\text { IDT }\end{array}$ & $\begin{array}{l}11.93(3.51) \\
11.75(2.87)\end{array}$ & $\begin{array}{l}11.54(3.20) \\
11.46(2.91)\end{array}$ & $\begin{array}{l}11.49(3.18) \\
11.30(3.12)\end{array}$ & $\begin{array}{l}11.23(3.24) \\
11.22(2.81)\end{array}$ & $.003^{*}$ & n.s. & n.s. & n.s. & n.s. & n.s. \\
\hline Step length asymmetry $(\mathrm{cm})$ & $\begin{array}{l}\text { CTT } \\
\text { IDT }\end{array}$ & $\begin{array}{l}3.84(5.09) \\
3.52(2.61)\end{array}$ & $\begin{array}{l}3.73(3.67) \\
3.47(2.82)\end{array}$ & $\begin{array}{l}3.67(4.28) \\
3.73(2.80)\end{array}$ & $\begin{array}{l}3.64(2.98) \\
3.45(2.03)\end{array}$ & n.s. & n.s. & n.s. & n.s. & n.s. & n.s. \\
\hline
\end{tabular}

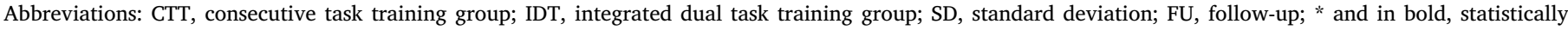

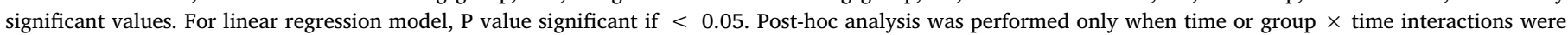
statistically significant. For post hoc analysis, P significant if $<.05$.

\section{Discussion}

In this secondary analysis of the DUALITY trial we compared the efficacy of two dual-task training programs in improving spatiotemporal outcome parameters other than gait velocity. We specifically focused on stride length, cadence, and gait variability because they are known to be impaired in PD and are relatively independent gait features $[1,2]$. Both training programs were equally effective in improving stride length and cadence. These effects were evident not only in the single-task condition but also transferred to dual-task conditions, and were maintained for up to 12 weeks after training. We found no significant changes in gait variability but only main effects of stride time under the auditory stroop task condition and stride length on the backward digit span task. This finding indicates that neither training modality significantly influenced variability in stride length and time.

The primary analysis of the DUALITY trial showed that gait velocity improved after a six-week program of integrated dual-task training (IDT) and consecutive task training (CCT) and was maintained for up to 12 weeks after training [10]. Improvement in gait velocity can result from increased cadence, increased stride length, or both. The results of our secondary analysis indicate that stride length and cadence improved with both training modalities. Unsurprisingly, significant results were also observed for other spatiotemporal gait parameters that are closely related to gait velocity, stride length, and cadence. For example, we observed a significant decrease in stride time, stance time, and double-support percentage. In addition, these improvements in gait parameters were also seen in the functional mobile phone task condition (Tables 2 and 3, Supplementary Tables 1 and 2).

No significant changes in stride length and stride time variability were noted after training with both modalities. Gait variability is an important outcome measure, as it predicts falls in older adults and people with PD [3]. The reasons for the absence of a significant training effect on gait variability are threefold. First, the absence might simply be due to the fact that the training programs were not specifically designed to improve gait variability. While neither training program focused specifically on stride length or cadence, yet these parameters did improve. Second, standard deviations of the outcome measures on gait variability were large, which explains the absence of a significant difference. Third, our results suggest that gait variability is an aspect of gait that is largely independent of gait speed, cadence, and stride length, and that it is perhaps relatively more resistant to training. Indeed, neural control of gait variability and stride length is believed to involve distinct and possibly partly overlapping neural networks [3]. An increase in gait variability in PD is presumably related to basal ganglia dysfunction [3]. The basal ganglia are a key component of automatic gait control and are responsible for coordinating in a timely manner (motor cue production) the components of a motor plan [15]. As gait variability did not improve, the training modalities did not seem to exert an effect on motor cue production during gait. Previous studies have shown that gait variability can be trained to some extent in patients with PD [16-18]. One of these studies involved treadmill training, which might act as an external cue. Future studies are needed to evaluate whether dual-task training with a treadmill or another form of external cueing is able to improve gait variability.

Consistent with the primary analysis, we found no differences between CTT and IDT. Both training programs may have produced similar improvements in gait function by improving gait automatization and by reducing the competition for attentional resources among the tasks $[8,10]$. Additionally, the improvement in gait parameters in both groups may be a consequence of the improved ability to prioritize a 


\section{Single and dual-task, stride length $(\mathrm{cm})$}

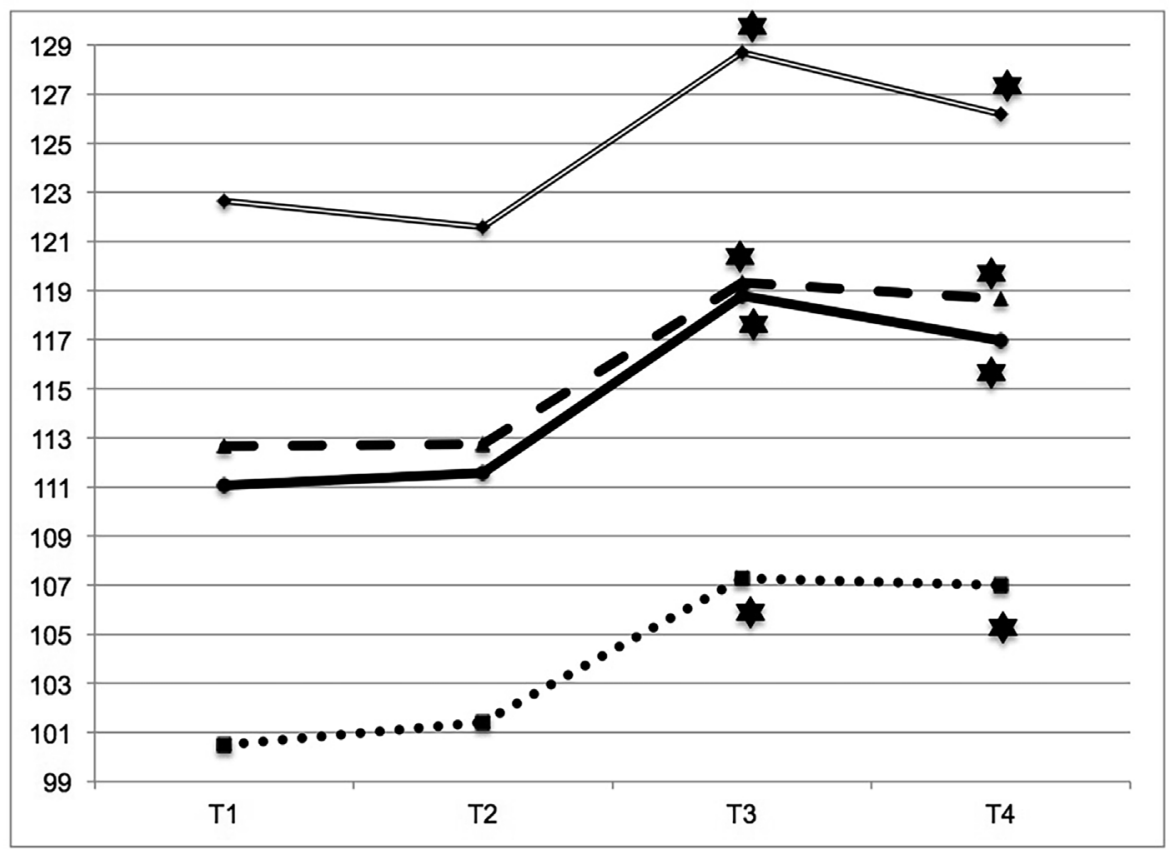

Single and dual-task, cadence (steps/min)

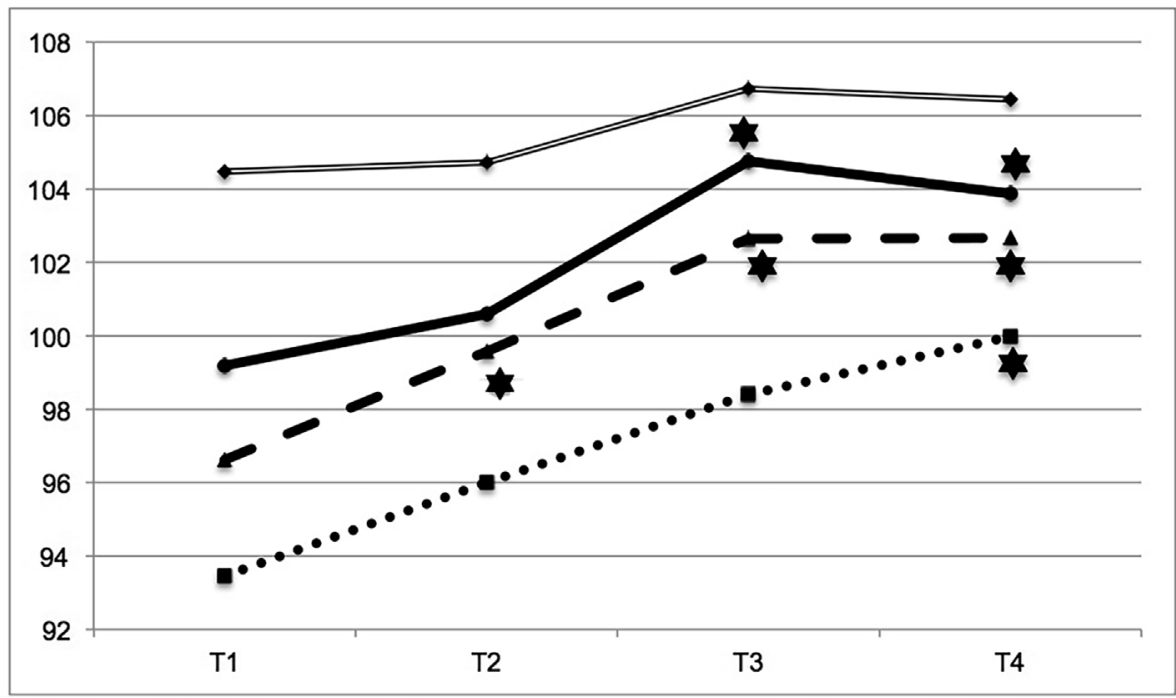

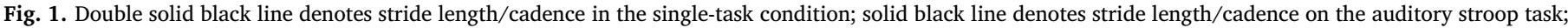

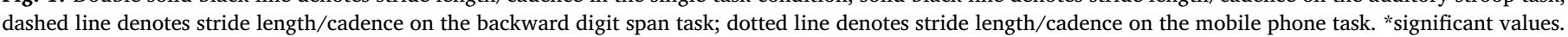

motor task over a cognitive one [19].

A limitation of this study is that LEDD was not stable over time, which may have biased our results, although this is not surprising as PD is a progressive disease. Levodopa is known to improve gait characteristics and gait variability [20,21]. As LEDD was not stable over time, and as it might have influenced the outcome measures, we introduced it as a covariate in our analysis.

Irrespective of its underlying mechanisms, the DUALITY study found that gait parameters during dual-tasking improved after both IDT and CCT in PD patients. These training modalities should be included among the rehabilitative approaches that physiotherapists use in their clinical practice.

\section{Financial disclosures}

The present study was financially supported by the Jacques and Gloria Gossweiler Foundation and the Malou Malou funds of the King Baudouin Foundation. The funding sources were not involved in study design or execution. All researchers involved in the study were independent of the funders.

\section{Conflicts of interest}

The Authors declare no conflicts of interest.

\section{Author roles}

Christian Geroin: data analysis and drafting the manuscript. 
Jorik Nonnekes: critical review.

Nienke M. de Vries: data collection and critical review.

Carolien Strouwen: data collection and critical review.

Nicola Smania: critical review.

Michele Tinazzi: critical review.

Alice Nieuwboer: study conception and design. Critical review.

Bastiaan R. Bloem: study conception and design. Critical review.

\section{Acknowledgments}

We would like to thank the patients for participating in this study.

\section{Appendix A. Supplementary data}

Supplementary data related to this article can be found at http://dx. doi.org/10.1016/j.parkreldis.2018.05.018.

\section{References}

[1] M.E. Morris, R. Iansek, T.A. Matyas, J.J. Summers, Stride length regulation in Parkinson's disease. Normalization strategies and underlying mechanisms, Brain 119 (1996) 551-568.

[2] S. Lord, B. Galna, L. Rochester, Moving forward on gait measurement: toward a more refined approach, Mov. Disord. 28 (2013) 1534-1543.

[3] J.M. Hausdorff, Gait dynamics in Parkinson's disease: common and distinct behavior among stride length, gait variability, and fractal-like scaling, Chaos 19 (2009) 026113.

[4] V.E. Kelly, A.J. Eusterbrock, A. Shumway-Cook, A review of dual-task walking deficits in people with Parkinson's disease: motor and cognitive contributions, mechanisms, and clinical implications, Parkinsons Dis. 2012 (2012) 918719.

[5] B.R. Bloem, V.V. Valkenburg, M. Slabbekoorn, M.D. Willemsen, The Multiple Tasks Test: development and normal strategies, Gait Posture 14 (2001) 191-202.

[6] J. Nonnekes, A.H. Snijders, J.G. Nutt, G. Deuschl, N. Giladi, B.R. Bloem, Freezing of gait: a practical approach to management, Lancet Neurol. 14 (2015) 768-778.

[7] Y.A. Grimbergen, M. Munneke, B.R. Bloem, Falls in Parkinson's disease, Curr. Opin. Neurol. 17 (2004) 405-415.

[8] L. Rochester, V. Hetherington, D. Jones, A. Nieuwboer, A.M. Willems, G. Kwakkel,
E. Van Wegen, Attending to the task: interference effects of functional tasks on walking in Parkinson's disease and the roles of cognition, depression, fatigue, and balance, Arch. Phys. Med. Rehabil. 85 (2004) 1578-1585.

[9] C. Strouwen, E.A. Molenaar, S.H. Keus, L. Münks, M. Munneke, W. Vandenberghe, B.R. Bloem, A. Nieuwboer, Protocol for a randomized comparison of integrated versus consecutive dual task practice in Parkinson's disease: the DUALITY trial, BMC Neurol. 14 (2014) 61.

[10] C. Strouwen, E.A.L.M. Molenaar, L. Münks, S.H.J. Keus, J.C.M. Zijlmans, W. Vandenberghe, B.R. Bloem, A. Nieuwboer, Training dual tasks together or apart in Parkinson's disease: results from the DUALITY trial, Mov. Disord. 32 (2017) 1201-1210.

[11] A.J. Hughes, S.E. Daniel, L. Kilford, A.J. Lees, Accuracy of clinical diagnosis of idiopathic Parkinson's disease: a clinico-pathological study of 100 cases, J. Neurol. Neurosurg. Psychiatry 55 (1992) 181-184.

[12] M.M. Hoehn, M.D. Yahr, Parkinsonism: onset, progression and mortality, Neurology 17 (1967) 427-442.

[13] T.N. Tombaugh, N.J. McIntyre, The mini-mental state examination: a comprehensive review, J. Am. Geriatr. Soc. 40 (1992) 922-935.

[14] B. Galna, S. Lord, L. Rochester, Is gait variability reliable in older adults and Parkinson's disease? Towards an optimal testing protocol, Gait Posture 37 (2013) $580-585$.

[15] J.G. Nutt, B.R. Bloem, N. Giladi, M. Hallett, F.B. Horak, A. Nieuwboer, Freezing of gait: moving forward on a mysterious clinical phenomenon, Lancet Neurol. 10 (2011) 734-744.

[16] S.G. Brauer, M.E. Morris, Can people with Parkinson's disease improve dual tasking when walking? Gait Posture 31 (2010) 229-233.

[17] G. Yogev-Seligmann, N. Giladi, M. Brozgol, J.M. Hausdorff, A training program to improve gait while dual tasking in patients with Parkinson's disease: a pilot study, Arch. Phys. Med. Rehabil. 93 (2012) 176-181.

[18] A. Mirelman, I. Maidan, T. Herman, J.E. Deutsch, N. Giladi, J.M. Hausdorff, Virtual reality for gait training: can it induce motor learning to enhance complex walking and reduce fall risk in patients with Parkinson's disease? J. Gerontol. A Biol. Sci. Med. Sci. 66 (2011) 234-240.

[19] B.R. Bloem, Y.A. Grimbergen, J.G. van Dijk, M. Munneke, The "posture second" strategy: a review of wrong priorities in Parkinson's disease, J. Neurol. Sci. 248 (2006) 196-204.

[20] M.S. Bryant, D.H. Rintala, J.G. Hou, E.C. Lai, E.J. Protas, Effects of levodopa on forward and backward gait patterns in persons with Parkinson's disease, NeuroRehabilitation 29 (2011) 247-252.

[21] M.S. Bryant, D.H. Rintala, J.G. Hou, R.L. Collins, E.J. Protas, Gait variability in Parkinson's disease: levodopa and walking direction, Acta Neurol. Scand. 134 (2016) 83-86. 